\title{
On (gg)*- Closed Sets in Topological Spaces
}

\author{
I. Christal Bai ${ }^{*}$, T.Shyla Isac Mary ${ }^{2}$ \\ ${ }^{1,2}$ Department of Mathematics, Nesamony Memorial Christian College, Marthandam, Tamil Nadu, India., \\ (Manonmaniam Sundaranar University, Abishekapatti, Tirunelveli, TamilNadu, India) \\ *Corresponding author: christalstalin@gmail.com
}

Available online at: www.isroset.org

Accepted 18/Aug/2018, Online 30/Aug/2018

\begin{abstract}
In this paper, we introduce a new class of closed sets called generalization of generalized star closed sets (briefly $(\mathrm{gg})^{*}$ - closed) in topological Spaces. A subset A of a topological space $(\mathrm{X}, \tau)$ is called $(\mathrm{gg})^{*}$ - closed if U contains regular closure of A whenever $\mathrm{U}$ contains $\mathrm{A}$ and $\mathrm{U}$ is gg-open in $(\mathrm{X}, \tau)$. We studied the relation of this set with some of the other closed and generalized closed sets and some of the characteristics, of $(\mathrm{gg})^{*}$ - closed sets have been investigated and studied.
\end{abstract}

Keywords: (gg)* - closed set, gg - open, regular closure.

AMS classification 2010 : 54A05

\section{INTRODUCTION}

The concept of generalized closed sets [1] in Topological spaces was introduced by N. Levine in 1970. D. E. Cameron and M. Stone introduced regular semi open sets [2] and regular open sets [3] respectively. In 2017, Basavaraj M. Ittanagi and Govardhana Reddy introduced and studied generalization of generalized closed sets [4] in Topological spaces.

In this paper we introduce a new class of closed set called (gg)* - closed sets in Topological spaces. Section 1, gives the overall introduction to the paper, followed by section 2, where we recall some of the existing closed and open sets. Section 3 , provides us with the introduction to the concept of $(\mathrm{gg})^{*}$ - closed set. In section 4, the independency of (gg)* - closed sets with some of the existing closed and generalized closed sets are studied and its outcome is shown in the form of a diagram. In section 5, some of the properties of $(\mathrm{gg})^{*}$ - closed sets are studied, analyzed and proved; which leads to section 6 , the conclusion of the paper. After which, the references that were dealt with during the analyses are given at the end of the paper.

\section{PRELIMINARIES}

Throughout this paper $(X, \tau)$ represent the topological space on which no separation axioms are assumed unless otherwise mentioned. For a subset $\mathrm{A}$ of $\mathrm{X}$, the closure of $\mathrm{A}$ and interior of A are denoted by $c l$ (A) and int (A) respectively.

Definition 2.1 A subset A of a topological space $(X, \tau)$ is called a

(1) generalized - closed set (briefly g - closed) [1] if $c l$ (A) $\subseteq \mathrm{U}$ whenever $\mathrm{A} \subseteq \mathrm{U}$ and $\mathrm{U}$ is open in $\mathrm{X}$.

(2) regular semi open [2] if there is a regular open set $\mathrm{U}$ such that $\mathrm{U} \subseteq \mathrm{A} \subseteq \operatorname{cl}(\mathrm{U})$.

(3) regular open set [3] if $A=\operatorname{int}(\operatorname{cl}(\mathrm{A}))$ and a regular closed set if $c l(\operatorname{int}(\mathrm{A}))=\mathrm{A}$.

(4) generalization of generalized closed set (briefly gg-closed) [4] if $\operatorname{gcl}(\mathrm{A}) \subseteq \mathrm{U}$ whenever $\mathrm{A} \subseteq \mathrm{U}$ and $\mathrm{U}$ is regular semi - open in $\mathrm{X}$. 
(5) semi - open set [5] if $\mathrm{A} \subseteq \operatorname{cl}(\operatorname{int}(\mathrm{A}))$ and a semi - closed set if $\operatorname{int}(\operatorname{cl}(\mathrm{A})) \subseteq \mathrm{A}$.

(6) pre - open set [6] if A $\subseteq \operatorname{int}(\operatorname{cl}(\mathrm{A}))$ and pre - closed if $c l(\operatorname{int}(\mathrm{A})) \subseteq \mathrm{A}$.

(7) semi - pre open set [7] if $\mathrm{A} \subseteq \operatorname{cl}(\operatorname{int}(\operatorname{cl}(\mathrm{A})))$ and semi pre - closed if $\operatorname{int}(\operatorname{cl}(\operatorname{int}(\mathrm{A}))) \subseteq \mathrm{A}$.

(8) $\beta$-open set [7] if $\mathrm{A} \subseteq \operatorname{cl}(\operatorname{int}(\operatorname{cl}(\mathrm{A})))$, whenever $\mathrm{A} \subseteq \mathrm{U}$ and $\mathrm{U}$ is open in $\mathrm{X}$.

(9) $\alpha$ - open set $[8]$ if $\mathrm{A} \subseteq \operatorname{int}(\operatorname{cl}(\operatorname{int}(\mathrm{A})))$ and $\alpha$ - closed set if $\operatorname{cl}(\operatorname{int}(\operatorname{cl}(\mathrm{A}))) \subseteq \mathrm{A}$.

(10) $\mathrm{t}-\operatorname{set}[9] \operatorname{iff} \operatorname{int}(\mathrm{A})=\operatorname{int}(\operatorname{cl}(\mathrm{A}))$.

(11) generalized semi - pre closed (briefly gsp - closed) [10] if $\operatorname{spcl}(\mathrm{A}) \subseteq \mathrm{U}$ whenever $\mathrm{A} \subseteq \mathrm{U}$ and $\mathrm{U}$ is open in $\mathrm{X}$.

(12) generalized pre - closed set (briefly gp - closed) [11] if pcl(A) $\subseteq \mathrm{U}$ whenever $\mathrm{A} \subseteq \mathrm{U}$ and $\mathrm{U}$ is open in $\mathrm{X}$.

(13) generalized semi - closed set (briefly gs - closed) [12 ] if $\operatorname{scl}(\mathrm{A}) \subseteq \mathrm{U}$ whenever $\mathrm{A} \subseteq \mathrm{U}$ and $\mathrm{U}$ is open in $\mathrm{X}$.

(14) $\alpha$-generalized closed set (briefly $\alpha$ g-closed) [13] if $\alpha c l$ (A) $\subseteq$ U whenever $\mathrm{A} \subseteq \mathrm{U}$ and $\mathrm{U}$ is open in $\mathrm{X}$.

(15) regular generalized closed set (briefly rg - closed ) [14] if $c l$ (A) $\subseteq \mathrm{U}$ whenever $\mathrm{A} \subseteq \mathrm{U}$ and $\mathrm{U}$ is regular - open in $\mathrm{X}$.

(16) generalized pre - regular closed set (briefly gpr - closed) [15 ] if pcl (A) $\subseteq \mathrm{U}$ whenever $\mathrm{A} \subseteq \mathrm{U}$ and $\mathrm{U}$ is regular open in $\mathrm{X}$.

(17) generalized semi - pre regular - closed set (briefly gspr - closed) [16] if $\operatorname{spcl}(\mathrm{A}) \subseteq \mathrm{U}$ whenever $\mathrm{A} \subseteq \mathrm{U}$ and $\mathrm{U}$ is regular open in $\mathrm{X}$.

(18) generalized star pre closed (briefly g*p - closed ) [17 ] if pcl (A) $\subseteq \mathrm{U}$ whenever $\mathrm{A} \subseteq \mathrm{U}$ and $\mathrm{U}$ is $\mathrm{g}$-open in $\mathrm{X}$.

(19) weakly closed set (briefly w - closed) [18 ] if $c l$ (A) $\subseteq \mathrm{U}$ whenever A $\subseteq \mathrm{U}$ and U is semi- open in X.

(20) tgr - closed set [19] if $r c l(\mathrm{~A}) \subseteq \mathrm{U}$ whenever $\mathrm{A} \subseteq \mathrm{U}$ and $\mathrm{U}$ is a t -set.

(21) regular w-closed (briefly rw - closed) [20] if $c l(\mathrm{~A}) \subseteq \mathrm{U}$ whenever $\mathrm{A} \subseteq \mathrm{U}$ and $\mathrm{U}$ is regular semi - open in X.

(22) regular gereralized $\alpha$ - closed set (briefly $\operatorname{rg} \alpha$ - closed) [21] if $\alpha c l(\mathrm{~A}) \subseteq \mathrm{U}$ whenever $\mathrm{A} \subseteq \mathrm{U}$ and $\mathrm{U}$ is regular $\alpha$ - open in $\mathrm{X}$

(23) gereralized $\alpha$-closed set (briefly g $\alpha$-closed) [22] if $\alpha c l(\mathrm{~A}) \subseteq \mathrm{U}$ whenever $\mathrm{A} \subseteq \mathrm{U}$ and $\mathrm{U}$ is $\alpha$ - open in $\mathrm{X}$.

(24) Semi - generalized closed set (briefly sg - closed) [23] if $s c l$ (A) $\subseteq$ U whenever A $\subseteq$ U and $\mathrm{U}$ is semi open in $\mathrm{X}$. 
(25) $\mathrm{R} *$ - closed set [24] if $r c l(\mathrm{~A}) \subseteq \mathrm{U}$ whenever $\mathrm{A} \subseteq \mathrm{U}$ and $\mathrm{U}$ is regular semi - open in $\mathrm{X}$.

(26) $\mathrm{R}^{\#}$ - closed set [25] if $\operatorname{gcl}(\mathrm{A}) \subseteq \mathrm{U}$ whenever $\mathrm{A} \subseteq \mathrm{U}$ and $\mathrm{U}$ is $\mathrm{R}^{*}$-open in $\mathrm{X}$.

(27) $\beta g^{*}$ - closed set [26] if $\operatorname{gcl}(\mathrm{A}) \subseteq \mathrm{U}$ whenever $\mathrm{A} \subseteq \mathrm{U}$ and $\mathrm{U}$ is $\beta$ - open in $\mathrm{X}$.

(28) $\mathrm{r}^{\wedge} \mathrm{g}$ - closed set [27] if $\operatorname{gcl}(\mathrm{A}) \subseteq \mathrm{U}$ whenever $\mathrm{A} \subseteq \mathrm{U}$ and $\mathrm{U}$ is regular - open in X.

(29) $\mathrm{g}^{* *}$ - closed set [28] if $c l(\mathrm{~A}) \subseteq \mathrm{U}$ whenever $\mathrm{A} \subseteq \mathrm{U}$ and $\mathrm{U}$ is $\mathrm{g} *$ - open in $\mathrm{X}$.

(30) $\mathrm{g}^{*}$ - closed set [29] if $\operatorname{cl}(\mathrm{A}) \subseteq \mathrm{U}$ whenever $\mathrm{A} \subseteq \mathrm{U}$ and $\mathrm{U}$ is $\mathrm{g}$ - open in $\mathrm{X}$.

(31) generalized regular closed set (briefly gr - closed) [30] if $r c l$ (A) $\subseteq$ U whenever $\mathrm{A} \subseteq \mathrm{U}$ and $\mathrm{U}$ is open in $\mathrm{X}$.

(32) generalized regular star closed (briefly gr *- closed) [31] if $r c l$ (A) $\subseteq$ U whenever $\mathrm{A} \subseteq \mathrm{U}$ and $\mathrm{U}$ is $\mathrm{g}$ - open in $\mathrm{X}$.

The complements of the above closed sets are their open sets and vice versa.

Definition 2.2 [19] The regular closure of a subset $\mathrm{A} \subseteq \mathrm{X}$ is the set $\operatorname{rcl}(\mathrm{A})=\cap\{\mathrm{B} \subseteq \mathrm{X}: \mathrm{B}$ is regular closed and $\mathrm{A} \subseteq$ B \}

\section{GENERALIZATION OF GENERALIZED STAR - CLOSED SETS}

Definition 3.1 A subset A of a topological space $(\mathrm{X}, \tau)$ is called generalization of generalized star closed sets (briefly (gg)*closed) if $r c l(\mathrm{~A}) \subseteq \mathrm{U}$ whenever $\mathrm{A} \subseteq \mathrm{U}$ and $\mathrm{U}$ is gg - open.

Example 3.2 Let $X=\{a, b, c, d\}$, and $\tau=\{\varphi,\{c\},\{d\},\{c, d\},\{a, c, d\}, X\}$ gg - open $=\{\varphi,\{a, c, d\},\{c, d\},\{a, d\},\{a, c\},\{a, b\},\{d\},\{b\},\{c\},\{a\}, X\}$ $(g g)^{*}-\operatorname{closed}=\{\varphi,\{a, b\},\{b, c\},\{b, d\},\{a, b, c\},\{a, b, d\},\{b, c, d\}, X\}$.

Proposition 3.3 Every regular closed set is $(\mathrm{gg}) *$ - closed.

Proof: Let $\mathrm{A}$ be a regular closed set in $\mathrm{X}$ such that $\mathrm{A} \subseteq \mathrm{U}$ and $\mathrm{U}$ is $g g$ - open. Then $\operatorname{rcl}(\mathrm{A})=\mathrm{A}$.

Hence $r c l(\mathrm{~A}) \subseteq \mathrm{U}$. Therefore $\mathrm{A}$ is $(\mathrm{gg}) *$ - closed.

Remark 3.4 The converse of the above proposition need not be true as shown in the following example.

Example 3.5 Let $X=\{a, b, c, d\}$, and $\tau=\{\varphi,\{c\},\{d\},\{c, d\},\{a, c, d\}, X\}$

Then the set $\{\mathrm{a}, \mathrm{b}\}$ is $(\mathrm{gg})^{*}$ - closed but not regular closed.

\section{Proposition 3.6}

(1) Every $(\mathrm{gg})^{*}$ - closed set is g- closed.

(2) Every $(\mathrm{gg}) *$ - closed set is $\mathrm{g} * *$-closed.

(3) Every (gg)* - closed set is gsp- closed.

(4) Every (gg)* - closed set is gp- closed.

(5) Every (gg)* - closed set is gs- closed. 
(6) Every (gg)* - closed set is $\alpha \mathrm{g}$ - closed.

(7) Every (gg)* - closed set is rg- closed.

(8) Every (gg)* - closed set is gpr- closed.

(9) Every (gg)* - closed set is gspr-closed.

(10) Every (gg)* - closed set is $\mathrm{g}^{*} \mathrm{p}$ - closed.

(11) Every (gg)* - closed set is gr-closed.

(12) Every $(\mathrm{gg}) *$ - closed set is gr*-closed.

Proof:

(1) Let A be a (gg)* - closed set in X. Let $\mathrm{U}$ be an open set in $\mathrm{X}$ such that $\mathrm{A} \subseteq \mathrm{U}$. Since every open set is gg-open [4] and since $\mathrm{A}$ is $(\mathrm{gg}) *$ - closed, $r c l(\mathrm{~A}) \subseteq \mathrm{U}$. But we have $\operatorname{cl}(\mathrm{A}) \subseteq \operatorname{rcl}(\mathrm{A}) \subseteq \mathrm{U}$. Hence $\mathrm{A}$ is g-closed.

(2) Let A be a (gg)* - closed set in $\mathrm{X}$. Let $\mathrm{U}$ be a $\mathrm{g}^{*}$-open set in $\mathrm{X}$ such that $\mathrm{A} \subseteq \mathrm{U}$. Since every $\mathrm{g}^{*}$-open set is gg -open [4] and since A is $(\mathrm{gg})^{*}$-closed, $r c l(\mathrm{~A}) \subseteq \mathrm{U}$. But we have $c l(\mathrm{~A}) \subseteq \operatorname{rcl}(\mathrm{A}) \subseteq \mathrm{U}$. Hence $\mathrm{A}$ is $\mathrm{g}^{* *}$-closed.

(3) Let A be a (gg)* - closed set in X. Let $\mathrm{U}$ be an open set in $\mathrm{X}$ such that $\mathrm{A} \subseteq \mathrm{U}$. Since every open set is gg - open [4] and since A is (gg)*-closed, $r c l(\mathrm{~A}) \subseteq \mathrm{U}$. But we have $\operatorname{spcl}(\mathrm{A}) \subseteq \operatorname{rcl}(\mathrm{A}) \subseteq \mathrm{U}$. Hence $\mathrm{A}$ is gsp-closed

(4) Let A be a (gg)* - closed set in X. Let $\mathrm{U}$ be an open set in $\mathrm{X}$ such that $\mathrm{A} \subseteq \mathrm{U}$. Since every open set is gg-open [4] and since $\mathrm{A}$ is $(\mathrm{gg}) *$-closed, $r c l(\mathrm{~A}) \subseteq \mathrm{U}$. But we have $\operatorname{pcl}(\mathrm{A}) \subseteq \operatorname{rcl}(\mathrm{A}) \subseteq \mathrm{U}$. Hence $\mathrm{A}$ is gp-closed

(5) Let A be a (gg)* - closed set in $X$. Let $U$ be an open set in $X$ such that $A \subseteq U$. Since every open set is $g g$-open [4] and since A is $(\mathrm{gg}) *$-closed, $r c l(\mathrm{~A}) \subseteq \mathrm{U}$. But we have $\operatorname{scl}(\mathrm{A}) \subseteq \operatorname{rcl}(\mathrm{A}) \subseteq \mathrm{U}$. Hence A is gs-closed.

(6) Let A be a (gg)* - closed set in $X$. Let $U$ be an open set in $X$ such that $A \subseteq U$. Since every open set is gg -open [4] and since A is (gg)*-closed, $r c l(\mathrm{~A}) \subseteq \mathrm{U}$. But we have $\alpha c l(\mathrm{~A}) \subseteq r c l(\mathrm{~A}) \subseteq \mathrm{U}$. Hence $\alpha c l(\mathrm{~A}) \subseteq \mathrm{U}$. Hence $\mathrm{A}$ is $\alpha$ g-closed.

(7) Let $\mathrm{A}$ be a (gg)* -closed set in $\mathrm{X}$. Let $\mathrm{U}$ be a regular open set in $\mathrm{X}$ such that $\mathrm{A} \subseteq \mathrm{U}$. Since every regular open set is $\mathrm{gg}$ - open [4] and $\mathrm{A}$ is $(\mathrm{gg})^{*}$ - closed, $r c l(\mathrm{~A}) \subseteq \mathrm{U}$. But we have $c l(\mathrm{~A}) \subseteq \operatorname{rcl}(\mathrm{A}) \subseteq \mathrm{U}$. Hence $\mathrm{A}$ is rg-closed.

(8) Let A be a (gg)* - closed set in $\mathrm{X}$. Let $\mathrm{U}$ be a regular open set in $\mathrm{X}$ such that $\mathrm{A} \subseteq \mathrm{U}$. Since every regular open set is gg - open [4] and $\mathrm{A}$ is $(\mathrm{gg}){ }^{*}$-closed, $r c l(\mathrm{~A}) \subseteq \mathrm{U}$. But we have $p c l(\mathrm{~A}) \subseteq \operatorname{rcl}(\mathrm{A}) \subseteq \mathrm{U}$. Hence $\mathrm{A}$ is gpr-closed.

(9) Let $\mathrm{A}$ be a (gg)* - closed set in $\mathrm{X}$. Let $\mathrm{U}$ be a regular open set in $\mathrm{X}$ such that $\mathrm{A} \subseteq \mathrm{U}$. Since every regular open set is gg - open [4] and $\mathrm{A}$ is $(\mathrm{gg}){ }^{*}$-closed, $r c l(\mathrm{~A}) \subseteq \mathrm{U}$. But we have $\operatorname{spcl}(\mathrm{A}) \subseteq \operatorname{rcl}(\mathrm{A}) \subseteq \mathrm{U}$. Hence $\mathrm{A}$ is gspr-closed

(10) Let A be a (gg)* - closed set in X. Let $\mathrm{U}$ be a g - open set in X such that $\mathrm{A} \subseteq \mathrm{U}$. Since every $\mathrm{g}$ - open set is gg - open [4] and $\mathrm{A}$ is $(\mathrm{gg}) *$-closed, $r c l(\mathrm{~A}) \subseteq \mathrm{U}$. But we have 
$\operatorname{pcl}(\mathrm{A}) \subseteq \operatorname{rcl}(\mathrm{A}) \subseteq \mathrm{U}$. Hence $\mathrm{A}$ is $\mathrm{g}^{*} \mathrm{p}$ - closed

(11) Let A be a (gg)* - closed set in X. Let $\mathrm{U}$ be an open set in $\mathrm{X}$ such that $\mathrm{A} \subseteq \mathrm{U}$.

Since every open set is gg- open [4] and since A is (gg)*-closed, $r c l(\mathrm{~A}) \subseteq \mathrm{U}$. Hence A

is gr- closed.

(12) Let $\mathrm{A}$ be a (gg)* - closed set in $\mathrm{X}$. Let $\mathrm{U}$ be a g- open set in $\mathrm{X}$ such that $\mathrm{A} \subseteq \mathrm{U}$.

Since every g- open set is gg- open [4] and since A is (gg)*-closed, $r c l(\mathrm{~A}) \subseteq \mathrm{U}$. Hence

A is gr*- closed.

Remark 3.7 The converse of the above proposition need not be true as shown in the following example.

Example 3.8 Let $X=\{a, b, c, d\}$, and $\tau=\{\varphi, \quad\{c\},\{d\},\{c, d\},\{a, c, d\}, X\}$. Then

(1) $\{\mathrm{b}\}$ is $\mathrm{g}$ - closed but not (gg)*-closed.

(2) $\{\mathrm{b}\}$ is $\mathrm{g}^{* *}$-closed but not $(\mathrm{gg}){ }^{*}$-closed.

(3) $\{$ a $\}$ is gsp-closed but not (gg)*-closed.

(4) $\{\mathrm{a}\}$ is gp-closed but not $(\mathrm{gg}) *$-closed.

(5) $\{\mathrm{d}\}$ is gs-closed but not $(\mathrm{gg}) *$-closed.

(6) $\{\mathrm{b}\}$ is $\alpha \mathrm{g}$-closed but not (gg)*-closed.

(7) $\{\mathrm{a}, \mathrm{c}\}$ is rg-closed but not $(\mathrm{gg}) *$-closed.

(8) $\{\mathrm{c}, \mathrm{d}\}$ is gpr-closed but not $(\mathrm{gg}) *$-closed.

(9) $\{\mathrm{a}, \mathrm{c}, \mathrm{d}\}$ is gspr-closed but not (gg)*-closed.

(10) $\{a\}$ is $g * p$ - closed but not $(\mathrm{gg}) *$-closed.

(11) $\{\mathrm{b}\}$ is gr-closed but not (gg)*-closed.

(12) $\{\mathrm{b}\}$ is gr*-closed but not $(\mathrm{gg}) *$-closed.

\section{INDEPENDENCY OF (gg)*-CLOSED SETS WITH OTHER CLOSED SETS.}

The following example shows that (gg)*-closed sets are independent from $\alpha$-closed, regular semi -closed, g $\alpha$-closed, rg $\alpha$ - closed, w -closed, rw- closed, sg - closed, pre - closed, g*s- closed, $\mathrm{R}^{*}$ - closed, tgr - closed.

\section{Example 4.1}

Let $X=\{a, b, c, d\}$, and $\tau=\{\varphi,\{c\},\{d\},\{c, d\},\{a, c, d\}, X\}$.Then

(1) $\{b, c\}$ is $(\mathrm{gg}) *$ - closed but not $\alpha$-closed and $\{b\}$ is $\alpha$-closed but not $(\mathrm{gg}) *$-closed.

(2) $\{\mathrm{b}, \mathrm{c}, \mathrm{d}\}$ is $(\mathrm{gg})^{*}$ - closed but not $\mathrm{g} \alpha$-closed and $\{\mathrm{a}\}$ is $\mathrm{g} \alpha$-closed but not $(\mathrm{gg})^{*}$ - closed.

(3) $\{\mathrm{a}, \mathrm{b}\}$ is $(\mathrm{gg}) *$ - closed but not regular semi - closed and $\{\mathrm{c}\}$ is regular semi -closed but not $(\mathrm{g} \mathrm{g}) *$ - closed. 
(4) $\{\mathrm{b}, \mathrm{c}\}$ is $(\mathrm{gg})^{*}$-closed but not $\operatorname{rg} \alpha$-closed and $\{\mathrm{a}, \mathrm{c}, \mathrm{d}\}$ is $\operatorname{rg} \alpha$-closed but not (gg)*-closed.

(5) $\{\mathrm{b}, \mathrm{d}\}$ is $(\mathrm{gg})^{*}$ - closed but not $\mathrm{w}$-closed and $\{\mathrm{b}\}$ is $\mathrm{w}$-closed but not $(\mathrm{gg}){ }^{*}$ - closed.

(6) $\{\mathrm{b}, \mathrm{c}\}$ is $(\mathrm{gg})^{*}$ - closed but not rw - closed and $\{\mathrm{c}, \mathrm{d})$ is rw - closed but not $(\mathrm{gg})^{*}$ - closed.

(7) $\{\mathrm{b}, \mathrm{c}, \mathrm{d}\}$ is $(\mathrm{gg}) *$ - closed but not sg - closed and $\{\mathrm{a}, \mathrm{d}\}$ is sg - closed but not (gg)*- closed.

(8) $\{\mathrm{b}, \mathrm{c}, \mathrm{d}\}$ is $(\mathrm{gg}) *$ - closed but not $\mathrm{g} * \mathrm{~s}$ - closed and $\{\mathrm{d}\}$ is $\mathrm{g} * \mathrm{~s}-$ closed but not $(\mathrm{gg}) *$ - closed.

(9) $\{\mathrm{a}, \mathrm{b}\}$ is $(\mathrm{gg})^{*}$ - closed but not pre- closed and $\{\mathrm{c}\}$ is pre- closed but not (gg)*- closed.

(10) $\{\mathrm{b}, \mathrm{d}\}$ is $(\mathrm{gg})^{*}$ - closed but not $\mathrm{R}^{*}$ - closed and $\{\mathrm{a}, \mathrm{c}, \mathrm{d}\}$ is $\mathrm{R}^{*}$ - closed but not $(\mathrm{gg})^{*}$ - closed.

(11) $\{\mathrm{b}, \mathrm{c}\}$ is $(\mathrm{gg})^{*}$ - closed but not tgr- closed and $\{\mathrm{c}, \mathrm{d}\}$ is tgr- closed but not $(\mathrm{gg})^{*}$ - closed.

\section{Remark 4.2}

From the above discussions and known results the relationship between (gg)*-closed sets and other existing generalizations of closed sets are implemented in Figure: 1

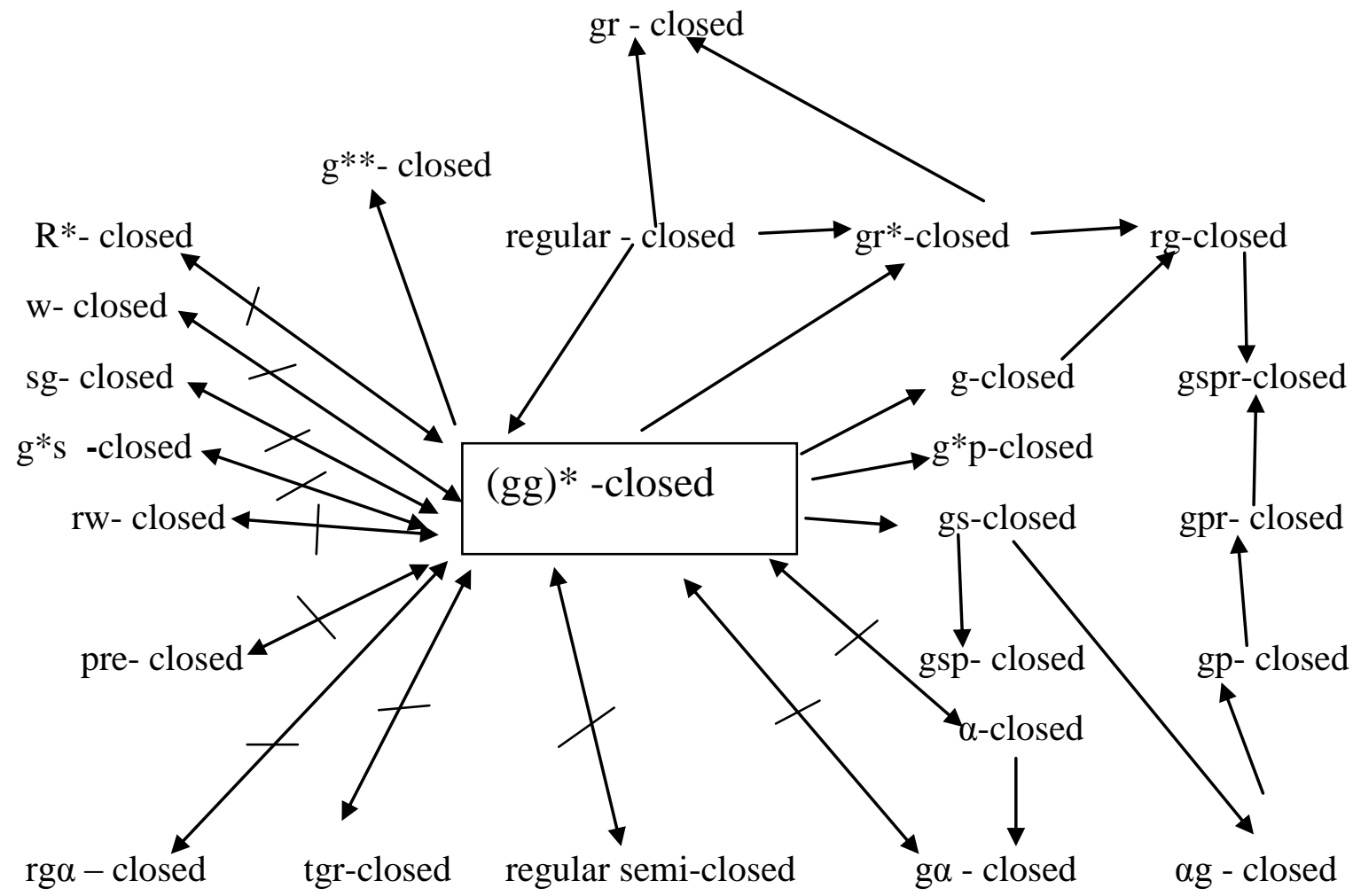

Figure: 1

In the above figure $\mathrm{A} \longrightarrow$ means the set $\mathrm{A}$ implies the set $\mathrm{B}$ but not conversely and $\mathrm{A} \longrightarrow$ means the set $\mathrm{A}$ and $\mathrm{B}$ are independent of each other. 


\section{CHARACTERISTICS OF (gg)* - CLOSED SETS}

Theorem 5.1 The Union of any two $(\mathrm{gg}) *$-closed sets of $\mathrm{X}$ is $(\mathrm{gg}) *$-closed.

Proof: Let $\mathrm{A}$ and $\mathrm{B}$ be the $(\mathrm{gg})^{*}$-closed sets in $\mathrm{X}$. Let $\mathrm{U}$ be a gg - open set in $\mathrm{X}$ such that $\mathrm{AU} \mathrm{B} \subseteq \mathrm{U}$. Then $\mathrm{A} \subseteq \mathrm{U}$ and $\mathrm{B} \subseteq \mathrm{U}$. Since $\mathrm{A}$ and $\mathrm{B}$ are $(\mathrm{gg})^{*}$-closed sets in $\mathrm{X}, r c l(\mathrm{~A}) \subseteq \mathrm{U}$ and $r c l(\mathrm{~B}) \subseteq \mathrm{U}$. We have by

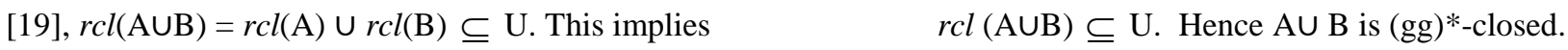

Remark 5.2 Intersection of two $(\mathrm{gg}) *$-closed sets need not be $(\mathrm{gg}) *$-closed as shown in the following example.

Example 5.3 Let $X=\{a, b, c, d\}$, and $\tau=\{\varphi,\{c\},\{d\},\{c, d\},\{a, c, d\}, X\}$.

Let $A=\{a, b\}$ and $B=\{b, c\}$ be two $(g g)^{*}$-closed sets in $X$

Then $\mathrm{A} \cap \mathrm{B}=\{\mathrm{b}\}$ is not $\mathrm{a}(\mathrm{gg}){ }^{*}$-closed set.

Theorem 5.4 A subset A of $\mathrm{X}$ is ( $\mathrm{gg})^{*}$-closed set in $\mathrm{X}$ if and only if $r c l(\mathrm{~A})$ - A contains no non-empty gg- closed set.

Proof : Let $\mathrm{F}$ be a non-empty gg-closed set in $\mathrm{X}$ such that $\mathrm{F} \subseteq \operatorname{rcl}(\mathrm{A})-\mathrm{A}$. That is

$\mathrm{F} \subseteq \operatorname{rcl}(\mathrm{A}) \cap\left[(\mathrm{A})^{\mathrm{c}}\right]$. Therefore $\mathrm{F} \subseteq \operatorname{rcl}(\mathrm{A})$ and $\mathrm{F} \subseteq \mathrm{A}^{\mathrm{c}}$ and so $\mathrm{A} \subseteq \mathrm{F}^{\mathrm{c}}$. Now since $\mathrm{A}$ is $(\mathrm{gg})^{*}$-closed, and $\mathrm{F}^{\mathrm{c}}$ is ggopen, $r c l(\mathrm{~A}) \subseteq \mathrm{F}^{\mathrm{c}}$. This implies $\mathrm{F} \subseteq[\operatorname{rcl}(\mathrm{A})]^{\mathrm{c}}$. Also we have $\mathrm{F} \subseteq \operatorname{rcl}(\mathrm{A})$. Therefore $\mathrm{F} \subseteq \operatorname{rcl}(\mathrm{A}) \cap[r c l(\mathrm{~A})]^{\mathrm{c}}=\varphi$. This is a contradiction. Therefore $\quad r c l(\mathrm{~A})-\mathrm{A}$ contains no non-empty gg-closed set.

Conversely, suppose that $\operatorname{rcl}(\mathrm{A})$ - A contains no non-empty gg- closed set. Suppose $\operatorname{rcl}(\mathrm{A})$ is not contained in U. Let $\mathrm{U}$ be a gg-open set in $\mathrm{X}$ such that $\mathrm{A} \subseteq \mathrm{U}$. Then $\operatorname{rcl}(\mathrm{A}) \cap \mathrm{U}^{\mathrm{c}}$ is a non - empty gg-closed set and contained in $\operatorname{rcl}(\mathrm{A})-\mathrm{A}$. Which is a contradiction. Hence $\mathrm{A}$ is a

$(\mathrm{gg})^{*}$-closed set.

Theorem 5.5 Let $\mathrm{A} \subseteq \mathrm{B} \subseteq \operatorname{rcl}(\mathrm{A})$ and $\mathrm{A}$ is (gg)*-closed set in $\mathrm{X}$, then $\mathrm{B}$ is also (gg)*-closed.

Proof: Let $\mathrm{U}$ be a gg-open set in $\mathrm{X}$ such that $\mathrm{A} \subseteq \mathrm{U}$. Now if $\mathrm{A} \subseteq \mathrm{B} \subseteq \operatorname{rcl}(\mathrm{A})$, then $r c l(\mathrm{~A}) \subseteq \operatorname{rcl}(\mathrm{B}) \subseteq \operatorname{rcl}(\mathrm{A})$. Therefore $r c l(\mathrm{~B})=\operatorname{rcl}(\mathrm{A})$. Since $\mathrm{A}$ is $(\mathrm{gg}) *$-closed, $r c l(\mathrm{~A}) \subseteq \mathrm{U}$.

Therefore $\operatorname{rcl}(\mathrm{B})=\operatorname{rcl}(\mathrm{A}) \subseteq \mathrm{U}$. Hence $\mathrm{B}$ is $(\mathrm{gg}) *$-closed.

Theorem 5.6 If A is gg-open subset of $\mathrm{X}$ and $(\mathrm{gg})^{*}$-closed set in $\mathrm{X}$. Then $\mathrm{A}$ is a regular closed set.

Proof: Since $\mathrm{A}$ is gg-open subset of $\mathrm{X}$ and $(\mathrm{gg})^{*}$ - closed, $\operatorname{rcl}(\mathrm{A}) \subseteq \mathrm{A}$. But $\mathrm{A} \subseteq \operatorname{rcl}(\mathrm{A})$.

Therefore $\mathrm{A}=\operatorname{rcl}(\mathrm{A})$. Hence $\mathrm{A}$ is regular closed.

Theorem 5.7 Let $\mathrm{A} \subseteq \mathrm{B} \subseteq \mathrm{X}$, where $\mathrm{B}$ is gg-open and (gg)*-closed in $\mathrm{X}$. If $\mathrm{A}$ is (gg)*-closed in B. Then A is (gg)*-closed in $X$.

Proof: Let $\mathrm{U}$ be a gg-open set in $\mathrm{X}$ such that $\mathrm{A} \subseteq \mathrm{U}$. Since $\mathrm{A} \subseteq \mathrm{U} \cap \mathrm{B}, \mathrm{U} \cap \mathrm{B}$ is gg-open in $\mathrm{B}$ and $\mathrm{A}$ is (gg)*-closed in B, $\operatorname{rcl}(\mathrm{A}) \subseteq \mathrm{U} \cap \mathrm{B}$. Now $r c l(\mathrm{~A}) \cap \mathrm{B} \subseteq \mathrm{U} \cap \mathrm{B}$. Since $\mathrm{A} \subseteq \mathrm{B}$,

$r c l(\mathrm{~A}) \subseteq \operatorname{rcl}(\mathrm{B})$. Since B is gg-open and $(\mathrm{gg})^{*}$ - closed in $\mathrm{X}$, by theorem 5.6, B is regular closed. Therefore $r c l(\mathrm{~B})=\mathrm{B}$. This implies $\operatorname{rcl}(\mathrm{A}) \subseteq \mathrm{B}$. Thus $r c l(\mathrm{~A})=\operatorname{rcl}(\mathrm{A}) \cap \mathrm{B} \subseteq \mathrm{U} \cap \mathrm{B} \subseteq \mathrm{U}$. Hence $\mathrm{A}$ is $(\mathrm{gg}) *$-closed in $\mathrm{X}$.

Theorem 5.8 For every point $x$ of the space $\mathrm{X}$ the set $\mathrm{X}-\{x\}$ is either (gg)*-closed (or) gg-open.

Proof: Suppose that $\mathrm{X}-\{x\}$ is not gg-open .Then $\mathrm{X}$ is the only gg-open set containing $\mathrm{X}-\{x\}$.

That is $\mathrm{X}-\{x\} \subseteq \mathrm{X}$. This implies $\operatorname{rcl}(\mathrm{X}-\{x\}) \subseteq \operatorname{rcl}(\mathrm{X}) \subseteq \mathrm{X}$. Therefore $\mathrm{X}-\{x\}$ is a

$(\mathrm{gg}) *$ - closed set in $\mathrm{X}$.

Theorem 5.9

(1) If $\mathrm{A}$ is $\beta$-open and $\beta \mathrm{g}^{*}$-closed set in $\mathrm{X}$. Then $\mathrm{A}$ is $(\mathrm{gg})^{*}$-closed.

(2) If $A$ is $R^{*}$-open and $R^{\#}$ - closed set in $X$. Then A is (gg)*-closed. 
(3) If A is regular open and $\mathrm{r}^{\wedge} \mathrm{g}$ - closed set in $\mathrm{X}$. Then $\mathrm{A}$ is (gg)*-closed.

\section{Proof:}

(1) Let $\mathrm{A}$ be a $\beta$-open and $\beta \mathrm{g}^{*}$-closed set in $\mathrm{X}$. Let $\mathrm{U}$ be any gg-open set in $\mathrm{X}$ such that $\mathrm{A} \subseteq \mathrm{U}$. By Definition 2.1(27), $g c l(\mathrm{~A}) \subseteq$ A. But we have $r c l(\mathrm{~A}) \subseteq g c l(\mathrm{~A}) \subseteq$ A. Therefore $r c l(\mathrm{~A}) \subseteq \mathrm{U}$. Thus we get $\mathrm{A}$ is $(\mathrm{gg})^{*}$-closed.

(2) Let $\mathrm{A}$ be a $\mathrm{R}^{*}$-open and $\mathrm{R}^{\#}$ - closed set in $\mathrm{X}$. Let $\mathrm{U}$ be any gg-open set in $\mathrm{X}$ such that $\mathrm{A} \subseteq \mathrm{U}$. By Definition 2.1(26), $g c l(\mathrm{~A}) \subseteq \mathrm{A}$. But we have $r c l(\mathrm{~A}) \subseteq g c l(\mathrm{~A}) \subseteq \mathrm{A}$. Hence $r c l(\mathrm{~A}) \subseteq \mathrm{U}$. Thus we get $\mathrm{A}$ is $(\mathrm{gg}) *$-closed.

(3) Let $\mathrm{A}$ be a regular open and $\mathrm{r}^{\wedge}$ g-closed set in $\mathrm{X}$. Let $\mathrm{U}$ be any gg-open set in $\mathrm{X}$ such that $\mathrm{A} \subseteq \mathrm{U}$. By Definition 2.1(28), $g c l(\mathrm{~A}) \subseteq \mathrm{A}$. But we have $r c l(\mathrm{~A}) \subseteq \operatorname{gcl}(\mathrm{A}) \subseteq \mathrm{A}$. Hence $r c l(\mathrm{~A}) \subseteq \mathrm{U}$. Thus we get $\mathrm{A}$ is $(\mathrm{gg}) *$ - closed.

\section{Theorem 5.10}

Let A be a regular semi-open set.

(a) If $\mathrm{A}$ is gg-closed then $\mathrm{A}$ is ( $\mathrm{gg}) *$-closed.

(b) If $\mathrm{A}$ is $\mathrm{R}^{*}$-closed then $\mathrm{A}$ is $(\mathrm{gg})^{*}$-closed.

\section{Proof:}

(a) Let $\mathrm{A}$ be a regular semi-open set and gg-closed set in $\mathrm{X}$. Let $\mathrm{U}$ be any gg-open set in $\mathrm{X}$ such that $\mathrm{A} \subseteq \mathrm{U}$. By Definition 2.1(4), gcl(A) $\subseteq \mathrm{A}$. But we have $r c l(\mathrm{~A}) \subseteq \operatorname{gcl}(\mathrm{A}) \subseteq \mathrm{A}$. Hence $r c l(\mathrm{~A}) \subseteq \mathrm{U}$. Thus we get $\mathrm{A}$ is $(\mathrm{gg})^{*}$-closed.

(b) Let $\mathrm{A}$ be a regular semi-open set and $\mathrm{R}^{*}$-closed set in $\mathrm{X}$. Let $\mathrm{U}$ be any gg-open set in $\mathrm{X}$ such that $\mathrm{A} \subseteq \mathrm{U}$. By Definition 2.1(25), $\operatorname{gcl}(\mathrm{A}) \subseteq \mathrm{A}$. But we have $r c l(\mathrm{~A}) \subseteq \operatorname{gcl}(\mathrm{A}) \subseteq \mathrm{A}$. Hence $r c l(\mathrm{~A}) \subseteq \mathrm{U}$. Thus we get $\mathrm{A}$ is $(\mathrm{gg}) *$-closed.

\section{CONCLUSION}

The class of $(\mathrm{gg})$ *-closed sets in topological spaces is defined using regular closure and gg-open sets .We have studied the relation of this set with some other closed sets and some of the properties are investigated.

\section{REFERENCES}

[1] N .Levine, Generalized closed sets in topology, Rend. Circ. Mat. Palermo, 2 , 89- 96, 1970.

[2] D. E. Cameron, Properties of s-closed spaces proc, Amer. Math. Soc., 72, 581-586,1978.

[3] M. Stone, Application of the theory of Boolean rings to general topology, Trans. Amer. MathSoci, 412, 374-481, 1937.

[4] Basavaraj M Ittanagi and Govardhana Reddy H G, On gg-Closed Sets in Topological Spaces, International Journal of Mathematical Archive8(8), 126-133, 2017.

[5] N. Levine, Semi-open sets and semi-continuity in topological spaces, Amer. Math. Monthly, 70, 36-41, 1963.

[6] A.S. Mashhour, M.E.Abd El-Monsef and S.N.El-Deeb, On pre-continious and weak pre- continious mappings, Proc. Math and Phys. Soc. Egypt ,53, 47-53,1982.

[7] D. Andrijevic, Semi-preopen sets, Mat. Vesnik 38 ,24-32, 1986.

[8] O. Njastad, On some classes of nearly open sets, Pacific J. Math., 15, 961-970, 1965.

[9] T.Indira and K. Rekha, Applications of *b-open sets and **b-open sets in Topological Spaces, Annals of pure and Applied Mathematics, 1, 4456,2012 .

[10] J. Dontchev, On generalizing semi-pre-open sets, Mem. Fac. Sci. Kochi Univ. ser. A. Math., $16,35-48,1995$.

[11] H. Maki, J. Umehara and T. Noiri, Generalized preclosed sets, Mem. Fac. Sci. Kochi Univ. Ser. A. Math, 17, 33-42, 1996.

[12] S.P. Arya and T.M.Nour, Characterizations of s-normal spaces, Indian J. Pure. Appl. Math.,21 (8), 717-719,1990.

[13] H. Maki, R. Devi and K. Balachandran, Associated topologies of generalized $\alpha$-closed sets and $\alpha$-generalized closed sets, Mem. Fac. sci. Kochi. Univ. Ser. A. Math.,15, 51-63, 1994.

[14 ] N. Palaniappan and K.C. Rao, Regular generalized closed sets, Kyungpook Math.J.,33, 211-219, 1993.

[15] Y. Gnanambal, On generalized pre regular closed sets in topological spaces, Indian J. Pure. Appl. Math., 28 (3),351-360,1997. 
[16] G.B Navalagi and Chandrashekarappa, On gspr closed sets in topological spaces, Vol.2,Nos 1-2, pp.35-44, 2010.

[17] M.K.R.S.Veerakumar, g*-preclosed sets, Acta Ciencia India, Vol.XXVIII M, 1, 51- 60, 2002.

[18] A. Pushpalatha, Studies on gereralizations of mappings in topological space, ph.D. Thesis, Bharathiar University Coimbatore, 2000.

[19] Ahmed A.El.Mabhouh and Hanady A.Al-Faiuomy, tgr-Closed Sets and t*gr-Closed Sets. International Mathematical Forum, Vol. 10, No. 5, $211-220,2015$.

[20] S.S. Benchalli and R.S.Walli, On RW-closed sets in topological spaces, Bull. Malaysian.Math. Sci.Soc., (2)30(2), 99-110, 2007.

[21] A.Vadivel and K.Vairamanickam, $\operatorname{rg} \alpha$-closed sets and $\operatorname{rg} \alpha$-open sets in topological Spaces, Int. Journal of Math. Analysis, 3 (37), 1803-1819, 2009

[22] H. Maki, R. Devi and K. Balachandran, generalized $\alpha$-closed sets in topology, Bull. Fukuoka Univ. Ed. Part (2), 42, 13-21, 1993.

[23] P. Bhattacharya and B.K. Lahiri, Semi-generalized closed sets in topological spaces, Indian J. Math, 29, 376-382, 1987.

[24] C. Janaki and Renu Thomas, On R* closed sets in topological spaces, International Journal of Mathematical Archive, 3(8), 3067-3074 , 2012.

[25] Basavaraj M Ittanagi and Raghavendra. K, On R\#-Closed Sets in Topological Spaces, International Journal of Mathematical Archive-8(8), 134$141,2017$.

[26] C. Dhanapakyam, k. Indirani, On $\beta g^{*}$ closed sets in topological Spaces, International journal of Applied Research ,2(4),388-391, 2016.

[27] D. Savithiri and C Janaki, On Regular^Generalized closed sets in topological spaces International Journal of Mathematical Archive, 4(4), 162$169,2013$.

[28] M. Pauline Mary Helen, Veronica Vijayan, Ponnuthai Selvarani, g** closed sets in Topological Spaces, IJMA, 3(5), 1-15, 2012.

[29] P. Sundaram and M. Sheik John, On w-closed sets in topology, Acta, Ciencia Indica, 4, 389-392, 2000.

[30] S. Bhattacharya, On Generalized Regular Closed Sets, Int. J.Contemp. Math. Sciences, 6, 145-152, 2011.

[31] K. Indirani, P.Sathishmohan and V. Rajendran, On gr*-Closed sets in a Topological Spaces, International journal of Mathematics trends and Technology, Vol, feb 2014.

\section{AUTHORS PROFILE}

I.Christal Bai is a Research scholar in Department of Mathematics, Nesamony Memorial Christian College, Marthandam, Tamil Nadu, India . She pursued her M.Sc., and M. Phil., from Manonmaniam Sundaranar University in 2008, and 2009. She has one year teaching experience as Lecturer in Pon Jesly College of Engineering, Nagercoil, India. Her area of interest is Topology.

T .Shyla Isac Mary pursued M. Sc., M.Phil., and Ph. D from Manonmaniam Sundaranar University in 1999, 2001 and 2012. She is currently working as Assistant Professor in Department of Mathematics from Nesamony Memorial Christian College, Marthandam, India since 2003. She has published 25 papers in reputed national and international journals. Her area of research is Topology. Under her guidance two scholars are awarded Ph.D and at present 5 scholars are doing Ph.D. She has 15 years of teaching experience.

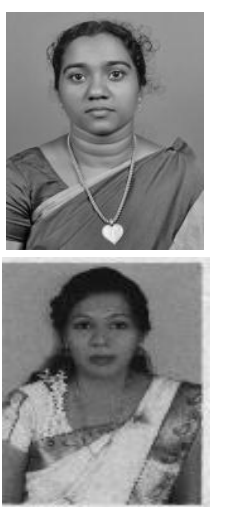

